\title{
Comparative Antigenic Analysis of Treponema pallidum Laboratory and Street Strains
}

\author{
By A. COCKAYNE, ${ }^{*}$ R. A. STRUGNELL, M. J. BAILEY† \\ AND C. W. PENN \\ Department of Biological Sciences, University of Birmingham, Birmingham B15 2TT, UK
}

(Received 16 February 1989; revised 26 April 1989; accepted 24 May 1989)

\begin{abstract}
The polypeptide and antigenic profiles of Treponema pallidum Nichols strain and two other more recently isolated 'street' strains of $T$. pallidum have been compared. PAGE and immunoblotting identified a $34.5 \mathrm{kDa}$ polypeptide present in the Nichols strain which was absent from one of the other street strains. This polypeptide was shown to be associated with the axial filament in $T$. pallidum. Three other axial-filament-associated polypeptides of 37,33 and $30 \mathrm{kDa}$ were present in all strains examined. Axial filaments of all three strains were morphologically identical and all three strains were equally motile.
\end{abstract}

\section{INTRODUCTION}

The vast majority of laboratory studies of Treponema pallidum have used the Nichols strain which was isolated in 1912 and has been maintained by passage in rabbits ever since (Turner \& Hollander, 1957). The strain remains virulent for the rabbit and man (Fitzgerald et al., 1976) but it is not known how closely related this strain is to the 'street' strains of $T$. pallidum which have been isolated more recently from infected individuals. Analysis of polypeptide and antigenic profiles of a limited number of other $T$. pallidum strains have revealed minor differences suggesting some limited genetic diversity amongst clinical isolates (Penn et al., 1986; Stamm \& Bassford, 1986). Analysis of such differences is important for a number of reasons. As the use of monospecific reagents is increased for the diagnosis and potential prophylaxis of syphilis it is important that antigens common to all $T$. pallidum strains are identified. In addition, analysis of such differences may allow an increased understanding of the genetic diversity and stability of isolates. This may be of great importance in considering potentially significant changes in the physiology of $T$. pallidum which may relate to changes in virulence or metabolic properties such as antibiotic resistance as recently reported by Stamm et al. (1988). Identification of differences between strains may also allow identification or elimination of treponemal components as virulence determinants, a procedure which in the past has been impossible because of the lack of an in vitro system for selection of suitable variants or mutants.

During analysis of a small number of relatively recent isolates of $T$. pallidum we identified one strain which lacked a major antigenic polypeptide present in the Nichols strain and another street strain. We report here on the identification of this polypeptide as a component of the axial filament of $T$. pallidum.

\section{METHODS}

Maintenance and growth of bacterial strains. Treponema pallidum subspecies pallidum, Nichols strain, was cultivated in the testes of New Zealand White rabbits as previously described (Penn \& Rhodes, 1982). Two other T. pallidum strains, designated street strains 1 and 2 , were obtained from Dr S. Lukehart, University of Washington, USA, and were cultivated in a similar manner. These strains were isolated in the USA from cases of

† Present address: NERC Institute of Virology, Mansfield Road, Oxford, UK. 
syphilis in 1983 and 1981 respectively. Prior to use bacteria were pelleted by centrifugation as described by Bailey et al. $(1987 a)$.

Small scale purification of axial filaments. Small quantities of axial filaments were prepared from $T$. pallidum as previously described (Cockayne et al., 1987) except that Sarkosyl washing of the sheared filaments was omitted.

SDS-PAGE and immunobloting. SDS-PAGE was done on $12.5 \%(\mathrm{w} / \mathrm{v})$ polyacrylamide gels essentially by the method of Laemmli (1970) as previously described (Cockayne et al., 1987). Two-dimensional (2-D) PAGE was done as previously described (Bailey et al., 1987a) but using a Bio-Rad Mini-2D electrophoresis system. Samples were solubilized by sonication in $9.2 \mathrm{M}$-urea, $8 \%(\mathrm{v} / \mathrm{v})$ Triton X-100 and $5 \%(\mathrm{v} / \mathrm{v}) \beta$-mercaptoethanol. Isoelectric focussing was done for $16 \mathrm{~h}$ at $200 \mathrm{~V}$ constant voltage and polypeptides then separated in the second dimension at $200 \mathrm{~V}$ constant voltage for $40 \mathrm{~min}$.

Polypeptides were transferred from SDS-PAGE gels to nitrocellulose membrane essentially by the method of Towbin et al. (1979) as previously described (Cockayne et al., 1987). Immunoblots were reacted with 1/200 dilution of antiserum or $1 / 20$ dilution of monoclonal antibody, for $2 \mathrm{~h}$ at room temperature prior to visualization of antibody binding as previously described (Cockayne et al., 1987).

Molecular masses of $T$. pallidum polypeptides are consensus values as reported by Norris $e t$ al. (1987). Lowmolecular-mass markers (Bio-Rad) were also electrophoresed on polyacrylamide gels for comparative purposes.

Antiserum and monoclonal antibodies. Polyclonal anti-T. pallidum Nichols strain antiserum was generated by repeated injection of sonicated bacteria into New Zealand White rabbits as previously described (Penn \& Rhodes, 1982).

Monoclonal anti-T. pallidum antibodies $\mathrm{CC} 9$, which recognizes the $37 \mathrm{kDa}$ axial-filament-associated polypeptide, and $\mathrm{SC} 12$ which reacts with three axial-filament-associated polypeptides of $34 \cdot 5,33$ and $30 \mathrm{kDa}$, were produced as previously described (Bailey et al., 1987b).

Electron microscopy. Treponemal preparations, resuspended in PBS, were transferred to Formvar-coated copper grids by floating the grids on drops of the preparations for $10 \mathrm{~min}$ at room temperature. Grids were washed briefly with distilled water and stained for $30 \mathrm{~s}$ with $3 \%(\mathrm{w} / \mathrm{v})$ sodium phosphotungstate, $\mathrm{pH} 7 \cdot 2$, in water, before viewing in a Philips 301 electron microscope.

\section{RESULTS}

\section{$P A G E$ analysis and immunoblotting}

Polypeptide profiles of $T$. pallidum Nichols strain and street strains 1 and 2, stained with PAGE Blue 83, are shown in Fig. 1 (a). The polypeptide profiles of all three strains were broadly similar, although a number of additional polypeptides, possibly reflecting variable levels of contamination of preparations with rabbit material, were present in both street strains. A weakly staining polypeptide of approximately $34.5 \mathrm{kDa}$, detected in the Nichols strain and in street strain 1, was reproducibly absent from street strain 2 (arrow). Immunoblots of similar preparations reacted with polyclonal anti-T, pallidum Nichols serum are shown in Fig. $1(b)$. All three strains were essentially antigenically identical except for the loss of a single antigenic polypeptide of $34.5 \mathrm{kDa}$ from street strain 2 .

\section{Purification of axial filaments}

The similarity in molecular mass of the $34.5 \mathrm{kDa}$ polypeptide with a polypeptide present in purified axial filaments of $T$. pallidum Nichols strain (Penn et al., 1985; Cockayne et al., 1987) prompted analysis of axial filaments from street strain 2 and Nichols strain organisms. Small quantities of axial filaments were therefore purified from the Nichols and street strain 2 . Immunoblots, reacted with polyclonal anti- $T$. pallidum Nichols strain serum, of purified preparations of axial filaments and whole cells from both strains are shown in Fig. 2(a). The Nichols strain axial filaments comprised four major antigenic polypeptides of molecular masses $37,34 \cdot 5,33$ and $30 \mathrm{kDa}$. These polypeptides were previously designated $\mathrm{j}, \mathrm{k}, \mathrm{l}$ and $\mathrm{m}$ by Norris $e t$ al. (1987). In contrast, axial filaments of street strain 2 lacked the $34.5 \mathrm{kDa}$ polypeptide. A number of other contaminating antigenic polypeptides, notably one of about $50 \mathrm{kDa}$, were also detected in these preparations.

The loss of the $34.5 \mathrm{kDa}$ polypeptide from street strain 2 was confirmed by reaction of Western blots of whole cells of both strains with monoclonal anti-axial-filament antibody SC12. This antibody recognized the $34 \cdot 5,33$ and $30 \mathrm{kDa}$ polypeptides in the Nichols strain, but only detected polypeptides of 33 and $30 \mathrm{kDa}$ in street strain 2 (Fig. 2b). The blot shown in Fig. 2(c) 
(a)

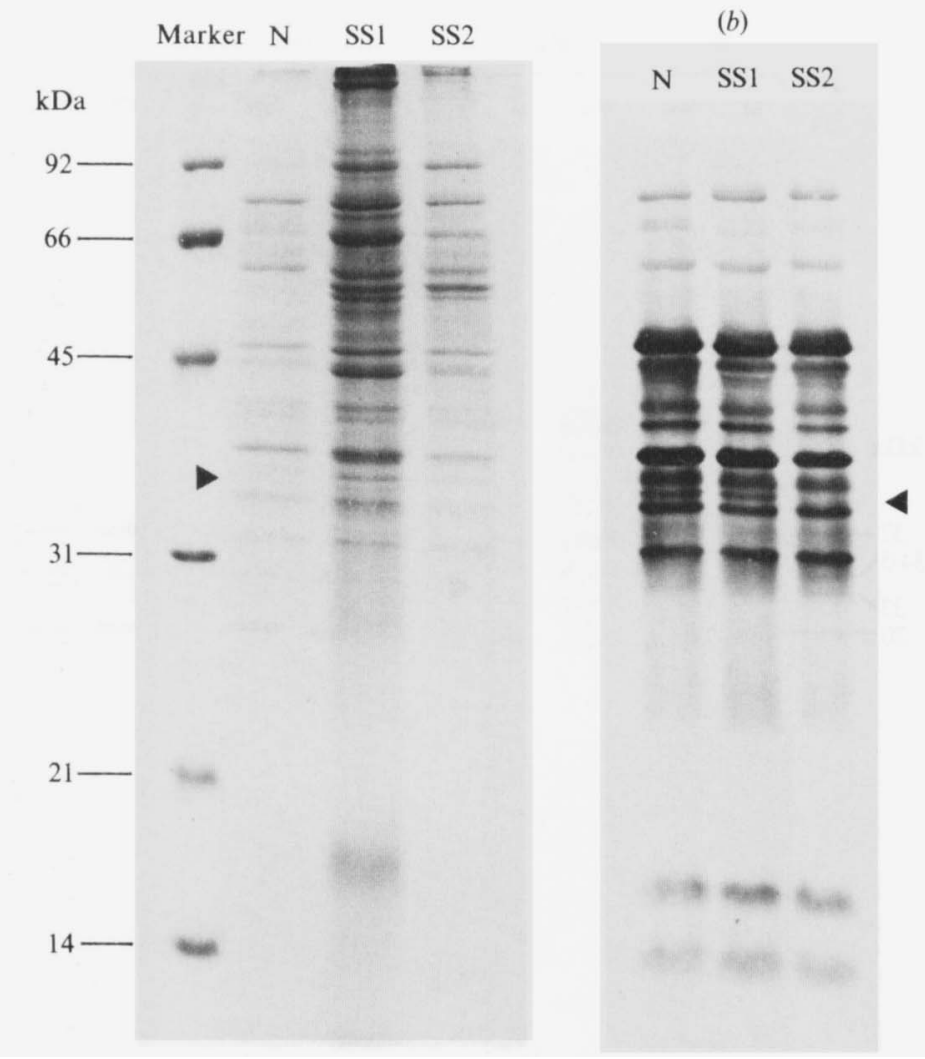

Fig. 1. SDS-PAGE (a) and immunoblot $(b)$ analyses of $T$. pallidum Nichols (N) and street strains (SS1 and SS2). Immunoblots were reacted with anti- $T$. pallidum Nichols strain rabbit serum.

was intentionally reacted with a mixture of monoclonal antibodies $\mathrm{CC} 9$ and $\mathrm{SC} 12$ to locate all four axial filament polypeptides simultaneously. The $37 \mathrm{kDa}$ axial filament polypeptide was recognized in both strains, presumably by monoclonal antibody CC9 (Fig. 2c).

\section{$2 D-P A G E$ and immunoblotting}

To investigate further any other potential differences between the two strains, preparations of whole cells and purified axial filaments were analysed by 2-D PAGE and immunoblotting. Immunoblotting of purified axial filaments of the Nichols strain identified the four spots associated with the axial filament (Fig. $3 b$ ). The $34.5 \mathrm{kDa}$ polypeptide spot was absent from 2-D immunoblots of street strain 2 (Fig. $3 d$ ). No other major differences between preparations of whole cells of the two strains were seen (Fig. $3 a, c$ ).

\section{Electron microscopy}

Electron micrographs of negatively stained treponemes are shown in Fig. 4. Axial filaments were released from treponemal cells by treatment with Triton X-100. The morphology of filaments from both the Nichols and street strain 2 was identical (Fig. $4 a, b$ ). The majority of axial filaments were sheathed although a small proportion of axial filaments had lost their sheath. As our previous results suggested that the $34.5 \mathrm{kDa}$ variable polypeptide was associated with the core of the axial filament (Cockayne et al., 1987), axial filaments from which the sheaths had been removed by treatment with trypsin and urea, were also examined. Removal of the sheath from both strains resulted in a decrease in the diameter and a marked loss of 


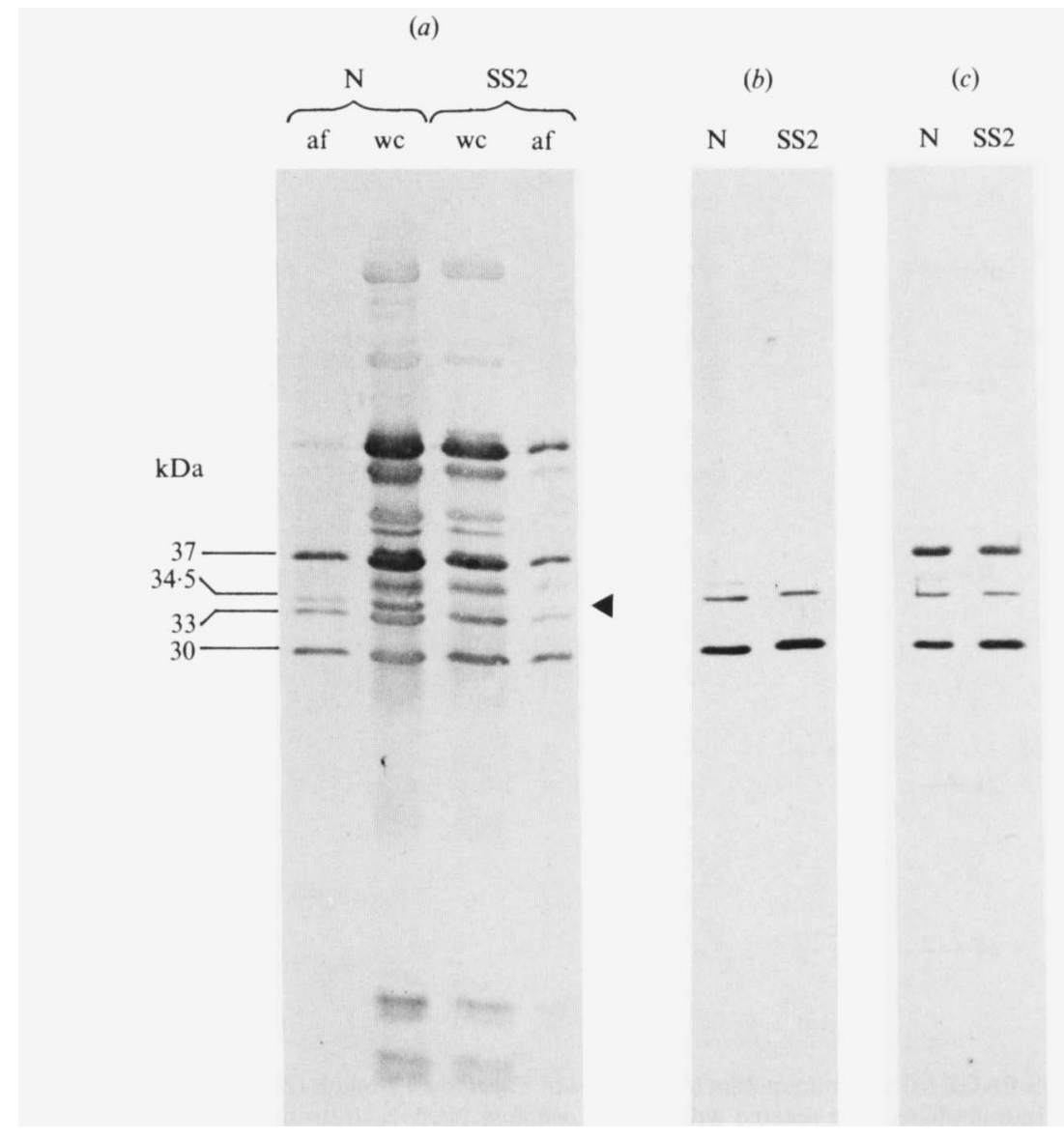

Fig. 2. (a) Immunoblot analysis of whole cells (wc) and purified axial filaments (af) of T. pallidum Nichols strain (N) and street strain 2 (SS2). Immunoblot reacted with polyclonal anti-T. pallidum rabbit serum. $(b, c)$ Immunoblot analysis of $T$. pallidum Nichols (N) or street strain 2 (SS2) whole cells reacted with anti- $T$. pallidum monoclonal antibodies. Immunoblots were reacted with monoclonal SC12 $(b)$ or with a mixture of monoclonals $\mathrm{SC1} 2$ and $\mathrm{CC} 9(c)$, to identify all four axial-filament-associated polypeptides.

curvature of the axial filaments (Fig. $4 c, d$ ). No apparent differences were observed in the morphology of the axial filament cores between the two strains (Fig. 4). The morphologies of intact axial filaments and core components of the axial filaments of street strain 1 were also examined and were found to be identical to those of the other two strains (data not shown).

\section{DISCUSSION}

Published comparisons between laboratory and street strains of $T$.pallidum are few, although two previous reports showed minor variations in the polypeptide profiles of $T$. pallidum Nichols strain and two more recent isolates (Penn et al., 1986; Stamm et al., 1988). No attempt was made to assign a function to the variable polypeptide in the first study: however, Stamm et al. (1988) were able to identify minor polypeptide differences between the Nichols strain and their street strain 14, which might correlate with the observed erythromycin resistance of this isolate. Our data, therefore, represent the first report of inter-strain variation in the polypeptide composition of a major structural component of $T$. pallidum, the axial filament.

Our previous experiments (Cockayne et al., 1987) showed that the 37 and $30 \mathrm{kDa}$ axial filament polypeptides of $T$. pallidum were solubilized by treatments which removed the axial 
(a) N-wc

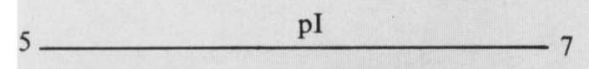

(c) $\mathrm{SS} 2-\mathrm{wc}$

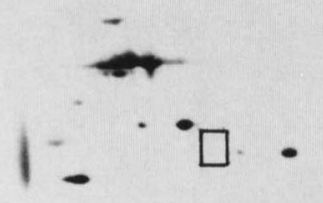

(b) $\mathrm{N}$-af

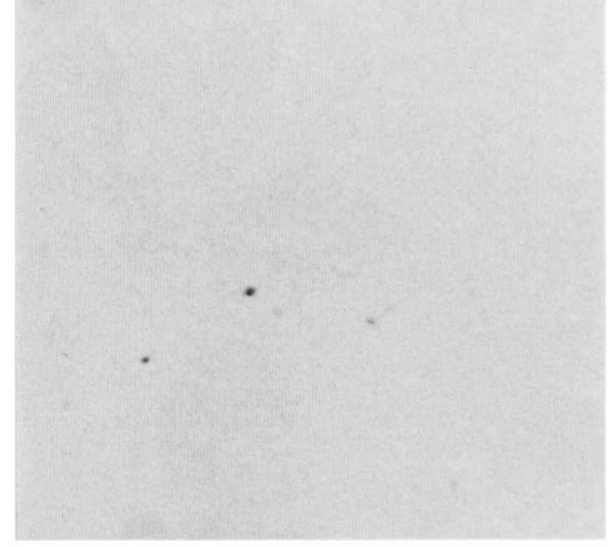

(d) SS2-af

Fig. 3. 2-D immunoblot analyses of whole cells (wc) or purified axial filaments (af) of Nichols (N) and street strain (SS2) isolates of $T$. pallidum. Immunoblots were reacted with anti-T. pallidum Nichols rabbit serum. Molecular masses of axial-filament-associated polypeptides are indicated on the Nichols whole cell immunoblot. The approximate $\mathrm{pI}$ range resolved in the first dimension is also indicated. The expected location of the missing $34.5 \mathrm{kDa}$ polypeptide spot in street strain 2 is indicated $(\square)$.

filament sheath. The axial filament core, consisting of the $34.5 \mathrm{kDa}$ variable polypeptide described here and a second of $33 \mathrm{kDa}$, was left morphologically intact. Both immunological (Limberger \& Charon, 1986) and.N-terminal amino acid sequence data (Norris et al., 1988) suggest that the structure of the core region of the axial filaments is highly conserved among treponemes, and may extend to the flagellins of other unrelated organisms (Norris et al., 1988). The loss of such a highly conserved polypeptide appears however, to have no observable effect on the motility of street strain 2 or on the morphology of the axial filaments of this strain. Norris et al. (1988) recently reported that the core-associated 34.5 and $33 \mathrm{kDa}$ polypeptides of the axial filaments of $T$. pallidum showed up to $95 \%$ homology at the amino acid level. Although these observations were based on limited amino acid sequence data (approximately 20 amino acids) the possibility exists that these two polypeptides may represent products of the same gene in $T$. pallidum which differ in the degree or type of post-translational modification. The possible structural relatedness of these two polypeptides, and in addition the $30 \mathrm{kDa}$ sheath-associated polypeptide, is supported by their antigenic relatedness as shown by reactivity of all three polypeptides with monoclonal antibody $\mathrm{SC1}$. The observation that treponemes lacking the 

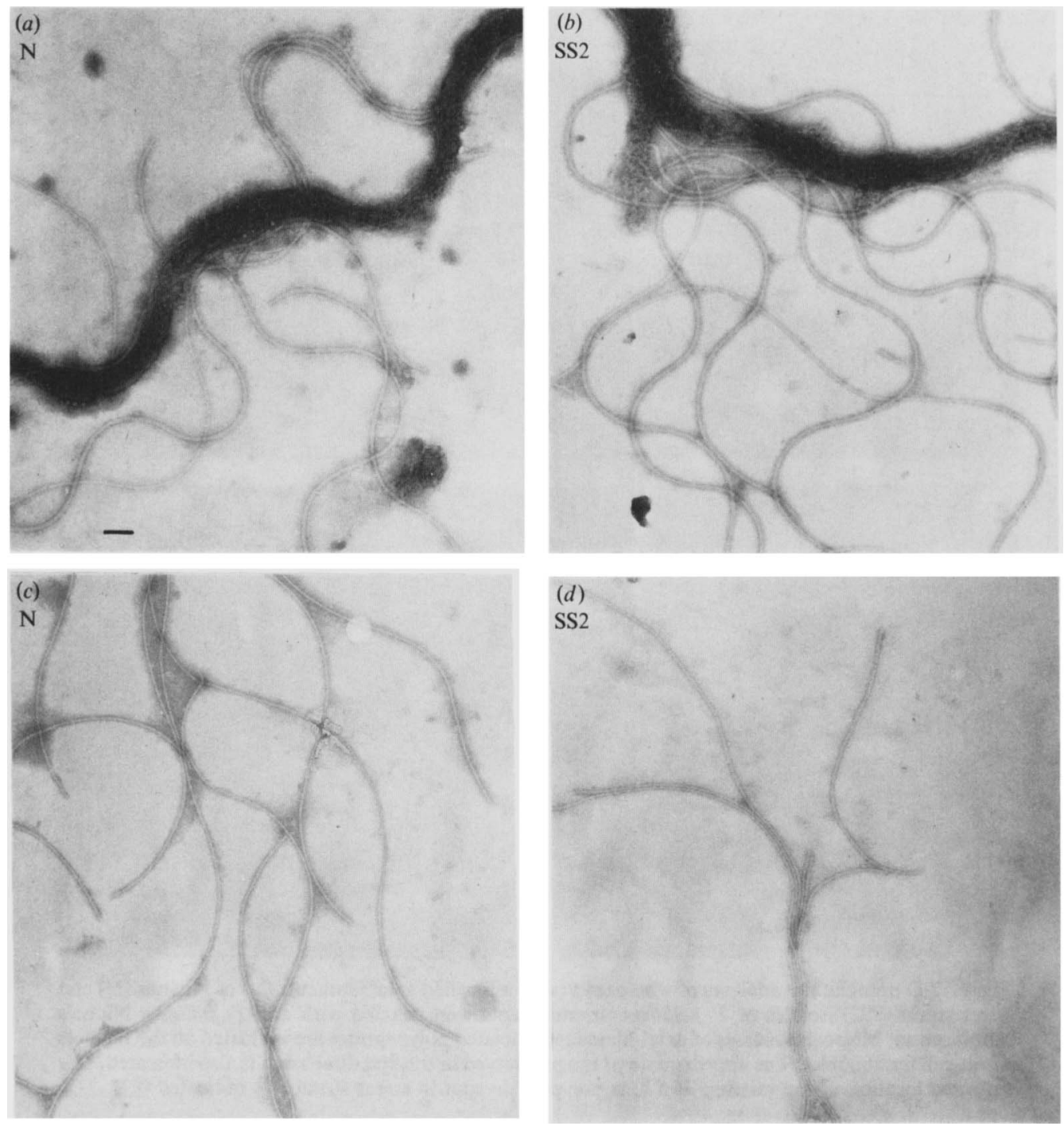

Fig. 4. Electron micrographs of negatively stained $T$. pallidum Nichols (N) and street strain 2 (SS2) treponemes. Bacteria were treated with either Triton X-100 alone $(a, b)$ or with Triton/trypsin and urea to expose the axial filament core structure $(c, d)$. Bar, $0 \cdot 1 \mu \mathrm{m}$.

$34.5 \mathrm{kDa}$ polypeptide show typical treponemal motility suggests that the $33 \mathrm{kDa}$ polypeptide represents the functional form in the axial filament core.

Of interest in all the strains examined was the effect of removal of the sheath in reducing the curvature of the axial filaments (Fig. 4). These observations suggest that the sheath-associated polypeptides are involved in the maintenance of the curvature of the axial filament shaft. The mechanism by which the shape of the intact axial filament shaft is maintained is unclear, but it may be envisaged that uneven tension along the length of the shaft could produce the characteristic curvature observed. The tension may be created by molecular interactions of sheath-associated polypeptides distributed unevenly along the length of the axial filaments. Further work to assess the detailed location of the various axial filament-associated polypeptides is required. To this end, molecular cloning of the genes encoding the axial filament polypeptides may allow analysis of the mechanisms of synthesis of these organelles in $T$. pallidum and provide 
further information on the interrelationships of the different axial filament polypeptides. These data may contribute to explaining the observed differences in the structure of spirochaetal axial filaments and the flagella of other bacteria. In addition, such studies would allow analysis of the nature of the mutation in street strain 2 , which results in the altered axial filament polypeptide profile we have detected.

The observed variation in the polypeptide and antigenic composition of different $T$. pallidum strains also has potential implications for the immunodiagnosis of syphilis. The use of recombinant antigens as immunodiagnostics is a natural step following cloning of $T$. pallidum genes in $E$. coli. The availability of recombinant $T$. pallidum polypeptides would alleviate problems associated with antigen currently produced by rabbit inoculation. However, our data and those from previous studies (Penn et al., 1986; Stamm et al., 1988) on the analysis of a very small number of street strains, suggest that care must be taken in the selection of antigens for use in such tests. Variation in treponemal structures as conserved as the axial filament suggests that a number of $T$. pallidum strains should be analysed to assess the usefulness of particular antigens. Existing reagents used in tests such as the TPHA test use a mixture of treponemal antigens and a similar approach using two or more recombinant antigens should reduce any potential problems associated with strain variation.

Thanks are due to Dr Sheila Lukehart for provision of the two street strains of $T$. pallidum. Thanks are also due to Mr S. H. A. Stevens and Mr D. Ruffles for excellent technical assistance.

\section{REFERENCES}

Bailey, M. J., Cockayne, A. \& Penn, C. W. (1987a). Monoclonal antibodies directed against surfaceassociated polypeptides of Treponema pallidum define a biologically active antigen. Journal of General Microbiology 133, 1793-1803.

Bailey, M. J., Cockayne, A. \& Penn, C. W. $(1987 \mathrm{~b})$. Production of murine monoclonal antibodies to the major axial polypeptide of Treponema pallidum. Journal of General Microbiology 133, 18051809.

Cockayne, A., Bailey, M. J. \& Penn, C. W. (1987). Analysis of sheath and core structures of the axial filament of Treponema pallidum. Journal of General Microbiology 133, 1397-1407.

Fitzgerald, J. J., Johnson, R. C. \& Smith, M. (1976). Accidental laboratory infection with Treponema pallidum, Nichols strain. Journal of the American Venereal Diseases Association 3, 76-79.

LAEMMLI, U. K. (1970). Cleavage of structural proteins during the assembly of the head of bacteriophage T4. Nature, London 227, 680-685.

Limberger, R. J. \& ChaRoN, N. W. (1986). Antiserum to the 33,000 Dalton periplasmic flagellum of Treponema phagedenis reacts with other treponemes and Spirochaeta aurantia. Journal of Bacteriology 168 , 1030-1034.

Norris, S. J., Alderete, J. F., Axelsen, N. H., Bailey, M. J., Baker-Zander, S. A., Baseman, J. B., Bassford, P. J., Baughn, R. E., Cockayne, A., Hanff, P. A., Hindersson, P., Larsen, S. A., LovetT, M. A., Lukehart, S. A., Miller, J. N., Moskophidis, M. A., Muller, F., Norgard, M. V., Penn, C. W., Stamm, L. V., Van Embden, J. D. \& WICHER, K. (1987). Identity of Treponema pallidum subsp. pallidum polypeptides: correlation of sodium dodecyl sulphate-polyacrylamide gel electrophoresis results from different laboratories. Electrophoresis 8, 77-92.
Norris, S. J., Charon, N. W., CoOK, R. J., Fuentes, M. D. \& Limberger, R. J. (1988). Antigenic relatedness and $\mathrm{N}$-terminal sequence homology define two classes of periplasmic flagellar proteins of Treponema pallidum subsp. pallidum and Treponema phagedenis. Journal of Bacteriology 170, 40724082.

Penn, C. W. \& Rhodes, J. G. (1982). Surface associated antigens of Treponema pallidum concealed by an inert outer layer. Immunology 46, 9-16.

Penn, C. W., Bailey, M. J. \& Cockayne, A. (1985). The axial filament antigen of Treponema pallidum. Immunology 54, 635-64I.

PenN, C. W., Bailey, M. J. \& Cockayne, A. (1986). Molecular and immunochemical analysis of Treponema pallidum. FEMS Microbiology Reviews 32, 139148.

Stamm, L. V. \& Bassford, P. J., JR (1986). Cellular and extracellular protein antigens of Treponema pallidum synthesized during in vitro incubation of freshly extracted organisms. Infection and Immunity 47, 799807.

Stamm, L. V., Stapleton, J. T. \& Bassford, P. J., JR (1988). In vitro assay to demonstrate high-level erythromycin resistance of a clinical isolate of Treponema pallidum. Antimicrobial Agents and Chemotherapy 32, 164-166.

Towbin, H., Staehelin, T. \& Gordon, J. (1979). Electrophoretic transfer of proteins from polyacrylamide gels to nitrocellulose sheets: procedure and some applications. Proceedings of the National Academy of Sciences of the United States of America 76, $4350-4354$.

TURner, T. B. \& Hollander, D. H. (1957). Biology of the Treponematoses. WHO Monograph series no. 35 . Geneva: WHO. 